\title{
Was ist Prominenz im Fernsehen?
}

\author{
Michael Niehaus
}

Das Phänomen der Prominenz lässt sich nicht zureichend erfassen, wenn man Prominenz als eine spezifische Eigenschaft versteht statt als Effekt von Zuschreibungen, der in der Regel einen Medienverbund voraussetzt. Wäbrend die Printmedien Prominenz zuschreiben, indem sie Leute zum Gegenstand von Diskursen machen, die bereits anderen Ortes Aufmerksamkeit gebündelt haben, ist das Fernseben in der Lage, Prominenz als Status zuzuerkennen. Erst im Fernseben erscheint die Prominenz als solche, da das Fernsehen nicht nur ein Medium, sondern zugleich ein Ort ist, an dem der Zuschreibungsakt der Prominenz vorgefübrt werden kann. Der Prominente wird als Prominenter angesprochen. Er wird als Gast begrüßt und anerkannt. Dadurch erhält er den Status des Prominenten, den er insofern nicht mehr einbüßen kann, als die Einladung im Prinzip stets wiederholbar ist. Von hier aus lässt sich unter anderem die Prominenz vom Rubm abgrenzen, da erstere die Möglichkeit medialer Präsenz voraussetzt und daher mit dem Tod erlischt. Weiterhin lassen sich vor diesem Hintergrund neuere Erscheinungsformen der Prominenz würdigen, die zum Beispiel die Überlagerung von Prominentenstatus und Kandidatenstatus in aktuellen Fernsebformaten betreffen.

Keywords: Prominenz, Medienprominenz, Prominenten-Status, Aufmerksamkeit, Fernsehgast, Berühmtheit, Elite

Die Frage, was Prominenz ist, scheint zu spät zu kommen. Schließlich ist die Prominenz in aller Munde und vor aller Augen. Es wird uns gesagt und gezeigt, wer prominent ist und wer nicht. Gleichwohl - oder vielleicht gerade deshalb - herrscht um den Begriff der Prominenz Einiges an Verwirrung und Missverständnis. Die folgenden Überlegungen sollen die Frage nach dem Wesen der Prominenz durch die nach den Formen ihrer Herstellung ersetzen. Es lässt sich beschreiben, wie Prominenz als Resultat von $\mathrm{Zu}$ schreibungen entsteht, die nur unter besonderen medialen Bedingungen erfolgen können und in ausgezeichneter Weise im Fernsehen vorgenommen werden. Um dies zu erläutern, möchte ich zunächst einige der bisherigen problemorientierten Bestimmungen des Prominenzbegriffs vorstellen (I), bevor ich mich anhand von Beispielen mit der logischen Erscheinungsform von Prominenz beschäftige (II), um die Ergebnisse abschließend für einen Blick auf neuere Entwicklungen des Prominenzphänomens im Fernsehen fruchtbar zu machen (III).

\section{Konzeptionen der Prominenz}

Das Wort „Prominenz“ leitet sich von dem lateinischen prominere ab, was „hervorragen“ oder „herausragen“ bedeutet. Dieser Hinweis findet sich fast überall, wo allgemeine Überlegungen zur Prominenz angestellt werden. Karl Kraus, ein „früher Zeuge der neuen Wortschöpfung “ (Peters 1996, S. 16), nannte - unter der Überschrift Prominente Pupperln - „prominent“ ein „gutes Fremdwort“, solange es „Seltenheitswert“ hatte, „denn man begnügte sich, jemand verdientermaßen ,hervorragend“ zu nennen“. Nun aber bezeichne das „grausliche Substantiv [...] keine Eigenschaft mehr, sondern eine Kategorie, eine Steuergruppe [...]: Die Prominenten, das sind die Obertanen“ (Kraus 1927/1961, S. 50). Der Prominente beißt also „der Hervorragende“, ist es aber nicht. Das 
„Hervorragend-Sein“ kann nämlich Kraus zufolge nicht als ein Titel gleichsam verlieben werden, weil es damit aufhört, eine Eigenschaft zu sein. Schon hier erscheint Prominenz also letztlich als Folge einer Zuschreibung.

Prominenz lässt sich daher nach Karl Kraus nicht absolut bestimmen, sondern nur relativ: Obertanen sind die, die keine Untertanen sind; die Prominenten unterscheiden sich von den Nicht-Prominenten. Entsprechend konstatiert Kraus eine Diffusion dieses „Ekelwort[es]“, das hauptsächlich „in den Spalten der Presse“ wuchere (und zwar vornehmlich in den Abendblättern, „die wenn's finster wird“ erscheinen): „Komödianten, Filmfritzen, Kabarettfatzken, Boxer, Fußballer, Parlamentarier, Eintänzer, Damenfriseure, Literaturhistoriker, Persönlichkeiten schlechtweg - alle können prominent sein“ (Kraus 1927/1961, S. 51). Prominente sind eben in einem wörtlichen Sinne genau „hervorragend“: Der „Genius der Sprache“, schreibt Gregor von Rezzori im vierten, Prominenz betitelten Teil seines Idiotenfübrers durch die deutsche Gesellschaft, habe den „sich Hervortuenden den Namen Prominenz gegeben - sachgerecht insofern, als damit keine absolute Größe und auch kein Wert bezeichnet ist, sondern lediglich der Abstand vom Niveau, von welchem sie sich jeweils hervortun" (Rezzori 1965, S. 7).

Prominenz ist also nicht nur etwas Relatives, sondern - wie schon Karl Kraus feststellte - damit zugleich auch etwas Unspezifisches. Man kann auf jede beliebige Weise prominent sein. Aber obwohl es - so Gregor von Rezzori - „zwischen so durchaus Verschiedenen keinerlei Gemeinsamkeiten geben kann, kraft deren sie soziologisch auf einen Nenner zu bringen wären - es sei denn eben in ihrem Status als Prominente", wirkten sie doch, „auch ohne Bindung und Zusammenschluss nach außen, als ob sie durchaus einer Art und Gattung, einer Bildung, einer Prägung wären." (Rezzori 1965, S. 13) Das Besondere an der Prominenz wäre demzufolge, dass sie zwar keine Eigenschaft bezeichnete, aber als eine solche aufgefasst würde. Etwa in diesem Sinne hatte schon Friedrich Sieburg formuliert, man müsse eigentlich sagen, „daß es die Prominenz eigentlich gar nicht gibt, daß sie also keine soziologisch umschreibbare Gruppe, sondern eine Vorstellung ist" (Sieburg 1954).

Den Definitionsversuchen von Prominenz haftet daher immer ein scheinhaftes oder tautologisches Moment an, das Birgit Peters in ihrer ausführlichen Untersuchung in dem Satz zusammenfasst: „Prominenz ist demnach berühmt, weil sie bekannt ist“ (Peters 1996, S. 20). Der Grund für diese Zirkularität ist in dem folgenden Definitionsansatz von Ralf Determeyer enthalten: „Auf jeden Fall impliziert ,hervorragen' ein Distanzmerkmal zu einer Mehrheit aufgrund von bestimmten Werten, die allgemeiner Anerkennung bedürfen, um eben ,hervorragend' zu sein" (Determeyer 1975, S. 165). Diese Anerkennung bezieht sich aber nicht auf eine besondere Leistung des Prominenten. Die Prominenz ist keine so genannte Leistungselite, sondern wird etwa als „Zufalls-Elite“ tituliert, bei der das „einzige Prinzip ihrer Exklusivität [...] im schlichten, faktischen Gegenteil jedes menschlich-verbindlichen Rangs" liege (Traugott 1979, S. 76). Insofern zollen wir den Prominenten unsere Anerkennung also zu Unrecht. Prominenz hat keinen zureichenden Grund, sondern entsteht letztlich aus dem Nichts. Sie erweckt aber den Anschein, als ob sie eine Elite wäre. Kann man daraus schließen, dass sie sich selbst mit der Elite verwechselt? Gefährlich ist es in jedem Falle, so Herfried Münkler, „wenn in einer Gesellschaft Prominenz und Elite fortgesetzt miteinander verwechselt werden“; Prominenz sei allenfalls so etwas wie ein „ins Grenzenlose aufgeblasenes hedonistisches Elitensegment, während die Elite selbst eher durch asketische Anforderungen geprägt ist" (Münkler 2004). Nun ist diese Entgegensetzung zweifellos ihrerseits elitär, und man kann einwenden, dass die Elite ohne weiteres ins Boot der Prominenz genommen wird - und sich ins Boot nehmen lässt unter der Voraussetzung einer Vereinbarkeit von Eli- 
te und Prominenz. Einem Beobachter kann das freilich als eine „Mutation der Elite zur Prominenz" im Zeitalter der Medien erscheinen (Macho 1993, S. 767).

In den bisherigen Bestimmungsversuchen stellt sich Prominenz letztlich als etwas Relatives, etwas Unspezifisches und etwas Grundloses dar. Prominent ist jemand, der im Vergleich zu den Anderen mehr oder weniger zufälliger Weise irgendwie hervorragt. Einer Antwort auf die Frage, warum das funktioniert, kann man näher kommen, wenn man eine Umkehrung vornimmt: Der Prominente bekommt keine Aufmerksamkeit, weil er eine Leistung erbringt, sondern seine Leistung besteht darin, Aufmerksamkeit zu bündeln. Aufmerksamkeit ist der Schlüsselbegriff der neueren Diskussion um die Prominenz - am markantesten vertreten durch Georg Francks Buch Ökonomie der Aufmerksamkeit. Dabei wird die Kategorie der Aufmerksamkeit zunächst quasi-anthropologisch fundiert: „Die Aufmerksamkeit anderer Menschen“ - so lautet ein viel zitierter Merksatz der Einleitung - „ist die unwiderstehlichste aller Drogen“ (Franck 1998, S. 10). Aus der „ökonomischen“ Perspektive einer letztlich messbaren Aufmerksamkeit erscheinen dann die Prominenten als „Einkommensmillionäre in Sachen Aufmerksamkeit“ (Franck 1998, S. 10). Zählbar ist der Reichtum an Aufmerksamkeit allerdings nicht durch die Menge der Augenpaare, die sich auf jemanden richten. Vielmehr gilt: „Wenn ein Prominenter Augen macht, dann reagiert das Zählwerk anders, als wenn irgend jemand schaut" (Franck 1998, S. 116). Denn Aufmerksamkeit regt Aufmerksamkeit an. Sie gleicht einem Kapital, das sich vermehrt.

Erlaubt die Beschreibung der Aufmerksamkeit in Termen der ökonomischen Analyse einen schärferen Blick auf das Funktionieren von Prominenz? Franck unterscheidet unter anderem den Rubm und die Prominenz als verschiedene Formen „rentierlichen Reichtums“. Der Ruhm ist ein „Vermögen in der Größenordnung“, das „ewige Rente“ verspricht, weil „auch diejenigen Beachtung schenken, die nicht genau wissen oder verstehen, wofür die Beachtung ursprünglich gezollt wurde“. Während der Ruhm ein alter Reichtum ist, bezeichnet die Prominenz eine Form neuen Reichtums. Die Prominenten sind „die klassischen Kapitalisten in der Ökonomie der Aufmerksamkeit“, von denen „allgemein bekannt ist, wer sie sind“, der „ursprüngliche Grund für die Bekanntheit“ hingegen „zweitrangig“ geworden ist: „Wie die Besitzer von Geldkapital, so beziehen Prominente auch dann noch ein Einkommen, wenn die Quellen des ursprünglichen Einkommens versiegt sind“ (Franck 1998, S. 118f.). Die Frage ist, wie weit eigentlich die Erkenntnis trägt, dass Aufmerksamkeit Aufmerksamkeit anregt, dass man sich irgendwie für die Prominenten interessiert, weil sie prominent sind usw. Eine Erklärung dafür, wie die gespendete Aufmerksamkeit dann doch zurückgehen kann, bietet diese Beschreibung jedenfalls nicht, und wenn man unter „Prominenz [...] die generalisierte Fähigkeit eines Akteurs“ versteht, „öffentliche Aufmerksamkeit zu finden“ (Gerhards/Neidhardt 1990, S. 36), so ist das kaum mehr als eine Worterklärung: Was hervorragt, erregt eben Aufmerksamkeit - und umgekehrt.

Man muss deshalb die bisher ausgeklammerte mediale Dimension von Aufmerksamkeit berücksichtigen. Dass Prominenz etwas mit Massenmedien zu tun hat, ist evident. Schließlich sollen sehr viele Menschen auf sehr wenige - eben „hervorragende“ - Menschen aufmerksam sein können. Darüber hinaus wird in den Prominenztheorien betont, dass die „wiederholte Sichtbarkeit einer Person“ (Peters 1996, S. 38; vgl. auch Peters 1993) für Prominenz konstitutiv sei. Prominente will man seben. Aber das allein ist noch keine Erklärung dafür, warum und vor allem in welcher Weise Sichtbarkeit für Prominenz - anders als für den Ruhm - unabdingbar ist. Auch im Radio können sehr viele Menschen sehr wenigen Menschen Aufmerksamkeit spenden. Aber prominent werden sie allein dadurch offenbar nicht - der durch den Internationalen Frühschoppen promi- 
nent gewordene Werner Höfer empfand es mit Hinblick auf seine Zeit beim Radio als „grotesk“, „daß Leute unserer Zunft erst dann zu Ansehen kamen, wenn man sie sehen konnte“ (zit. nach Peters 1996, S. 19).

Auch die Herkunft des Wortes Prominenz verweist ja auf die Sphäre des Sichtbaren. Das, was hervorragt, muss man eben sehen können. Und beim nächsten Hinschauen sollte es immer noch hervorragen. Von daher erscheint es als nicht weiter erklärungsbedürftig, dass das Fernsehen das adäquate Medium der Prominenz ist. Georg Franck handelt diesen Aspekt unter den Überschriften „Vom Publikations- zum Massenmedium“ und „Die Produktion der Prominenz“ ab. „Mit Presse, Rundfunk und Tonfilm kamen erstmals die für die Geburt von Stars kritischen Mengen anonym gespendeter Aufmerksamkeit zusammen“, aber erst „mit dem Fernsehen beginnt die zweite, eigens zur Attraktion von Aufmerksamkeit herausgebrachte Schauseite, der Wirklichkeit der ersten, unvermittelt angeschauten, Konkurrenz zu machen“ (Franck 1998, S. 147). Das Fernsehen bietet, wie es heißt, den in ihnen Auftretenden ein „Forum“ (Franck 1998, S. 148): „Der Auftritt im Fernsehen bedeutet die Chance, mit einem Schlag reich an Beachtung zu werden“ (Franck 1998, S. 150) und gegebenenfalls zu bleiben. Denn, so weiß Franck: „Prominent wird man heute durch standardisierte Karrieren. Am Anfang steht nicht mehr und nicht weniger, als irgendwie in die Medien zu finden. Weil es zunächst auf die Präsenz in den Medien ankommt, sollte der Auftritt am besten mit Bild und am allerbesten im Fernsehen erfolgen. Die Karriere nimmt ihre erste Hürde, wenn der Eindruck beim Publikum kommentiert, wenn der Auftritt besprochen wird“ (Franck 1998, S. 151).

Das ist eine eher entdifferenzierende Betrachtungsweise. Denn es ist natürlich keineswegs gleichgültig, in welcher Rolle der Auftritt im Fernsehen stattfindet. Wer beispielsweise als Wettkandidat bei Wetten, daß ...? die Aufmerksamkeit von Millionen einstreicht, sollte - wie Peter Lückemeier in seinem amüsanten Buch So werden Sie prominent feststellt - wissen, „dass die Chance gering ist, durch einen Auftritt in solchen Shows für eine größere Karriere entdeckt zu werden“ (Lückemeier 2003, 193). Die Wirkung des Mediums Fernsehen lässt sich nicht darauf reduzieren, dass es einen simultanen Multiplikationseffekt hat, dass es den Gegenstand der Aufmerksamkeit massenhaft überträgt und dadurch die Aufmerksamkeit vervielfacht. Der gerne zitierte Ausspruch (vgl. Peters 1996, S. 19f.) von Andy Warhol, dass heutzutage - also im Zeitalter der Massenmedien - jeder für fünfzehn Minuten berühmt sein könne, betrifft das Phänomen der Prominenz lediglich indirekt. Reduziert man die Funktion der Medien auf die Bereitstellung dieser Möglichkeit, so kann man auch ganz von ihnen absehen. So schreibt Birgit Peters etwa, die „Medien“ hätten „Prominenz nicht erfunden“, sondern es lediglich „mehr und anderen Personen“ erlaubt, „den Prominentenstatus zu erreichen“ (Peters 1996, S. 31f.), und als Beispiel führt sie Herostrat an, „der 359 v. Chr. einen Tempel in Ephesos anzündete, allein mit dem Ziel, berühmt zu werden“ (Peters 1996, S. 31).

Herostrat mag durch seine Tat eine - traurige - Berühmtheit erlangt haben, prominent geworden ist er durch sie nicht. Man wird nicht durch eine einzelne Tat oder durch ein einzelnes Ereignis prominent. Das wissen vor allem die Juristen, die vor der Frage stehen, wann das „Selbstdarstellungsrecht“ einer Person „von einem höherrangigen Allgemeininteresse verdrängt“ wird, nämlich vom „Informationsinteresse der Allgemeinheit" (Seemann 1996, S. 145). Sie unterscheiden in dieser Beziehung zwischen den relativen und den absoluten Personen der Zeitgeschichte. Erstere sind „durch die Verknüpfung mit einem Ereignis in das Blickfeld der Öffentlichkeit gelangt“ (Seemann 1996, S. 145), Letztere nehmen „zumindest für eine bestimmte Zeit kontinuierlich am öffentlichen Leben“ teil und begründen damit „unabhängig von Einzelereignissen ein andau- 
erndes Informationsinteresse der Öffentlichkeit“: „Dies sind in der Regel vor allem Prominente [...].“ (Ricker 1989, 106) Prominent sind also diejenigen, für die wir uns auch ohne Grund interessieren können. Und weiterhin kann dem Prominenten unterstellt werden, dass er auch am öffentlichen Leben teilnehmen will. Wie sollte sich der Prominente auf das Recht am eigenen Bild berufen können, „wenn er gleichzeitig das Licht der Öffentlichkeit sucht“ (Seemann 1996, 145)? Die Zirkularität braucht den Juristen nicht zu beunruhigen.

\section{Das Fernsehen erzeugt Prominenz als solche}

Es ist nicht verwunderlich, dass man in Schwierigkeiten kommt, wenn man Prominenz als wie auch immer geartete Eigenschaft auffasst. Schließlich ist es ja nicht er selbst, sondern unsere Aufmerksamkeit, die den Prominenten macht. Prominenz ist keine inhärente Eigenschaft. Das gilt auch für die Berühmtheit: Vincent van Gogh war zu Lebzeiten nicht berühmt. Jetzt ist er berühmt. Aber deswegen ist die Berühmtheit nicht zu einer Eigenschaft der Person Vincent van Gogh geworden. Sie hat sich nur an seinen $\mathrm{Na}-$ men geheftet. Das ist mehr oder weniger trivial. Das Merkwürdige ist nun, dass zwar auch der Prominenzbegriff offenbar keine inhärente Eigenschaft bezeichnet, aber gleichwohl völlig anders verwendet wird. Jürgen W. Möllemann beispielsweise ist das Paradebeispiel eines prominenten Politikers. Ganz unabhängig von der Frage, ob er derzeit auch berühmt ist, kann man vermuten, dass er es in hundert Jahren nicht mehr sein wird. Würde man aber sagen, dass er jetzt prominent ist? Man würde zweifellos eher sagen müssen, dass er ein prominenter Politiker war. Denn mit seinem Tod hat er im Grunde aufgehört, prominent zu sein. Er hat aufgehört, am öffentlichen Leben teilzunehmen.

Berühmtheit ist eine völlig andere Kategorie als Prominenz, und diese Verschiedenheit ist mit den von Georg Franck verwendeten Begriffen des alten und des neuen Reichtums nicht zureichend erfasst. Bleibt man bei der ökonomischen Terminologie, so bezeichnet Prominenz den Reichtum an Aufmerksamkeit, den es nur zu Lebzeiten gibt eine Art Leibrente. Wer nicht mehr zurückkommen kann, um sein Kapital an Aufmerksamkeit einzustreichen, der ist nicht mehr prominent - auch wenn ihm noch viel postume Aufmerksamkeit gewidmet wird (wenn ihm ehrende Nachrufe zuteil werden, wenn er Gegenstand von Lexikoneinträgen, Biographien oder Abhandlungen wird, wenn Straßen oder Stiftungen nach ihm benannt werden). Anders als der Ruhm ist die Prominenz also keine Eigenschaft, obwohl man gewissermaßen zeitgenössisch zu seiner eigenen Prominenz ist, obwohl sie einem hier und jetzt zugeschrieben wird. Daher lässt sie sich mit einer Eigenschaft verwechseln.

Prominent ist also jemand, der zurückkommen kann. Wohin zurück? Ins öffentliche Leben, ins öffentliche Bewusstsein. Aber was heißt das eigentlich? Für die meisten Leute heißt es gewiss: ins Fernsehen. Das liegt aber nicht einfach daran, dass das Fernsehen das am meisten frequentierte Massenmedium ist. Karl Kraus beispielsweise konstatierte das Grassieren von Prominenz schon zu einer Zeit, als es in der so genannten Medienlandschaft noch kein Fernsehen gab, und er verortete es „in den Spalten der Presse“, und noch Gregor von Rezzori bezieht sich 1965 auf die „Illustrierten“ als die primäre Quelle und Materialquelle von Prominenz. Dort wird über Leute geredet, die auf anderen Foren oder in anderen Medien Aufmerksamkeit erregt haben und sichtbar in Erscheinung getreten sind: etwa die „Komödianten“ auf der Kinoleinwand, die „Fußballer“ im Stadion oder die „Parlamentarier“ im Parlament.

Prominenz entsteht nicht dadurch, dass jemandem massenhaft Aufmerksamkeit ge- 
spendet wird, sondern dadurch, dass es ein Massenmedium gibt, in dem diese massenhafte Aufmerksamkeit besprochen wird, in dem sie zum Ausgangspunkt eines Diskurses wird, der sich dann mit den Leistungen, dem Privatleben oder der Lebensgeschichte des Betreffenden befassen kann. In diesem Sinne wird Prominenz zunächst einmal durch eine Art Medienverbund erzeugt. Damit ist zunächst einmal nichts weiter gemeint, als dass Prominenz nur entstehen kann, wo ein Medium in der Lage ist, die an einem anderen medialen Ort gespendete massenhafte Aufmerksamkeit zu thematisieren, zu kommentieren und dadurch zu verwandeln. Dies waren zunächst die Printmedien. In einem begrenzten Umfang können die Printmedien auch aus sich selbst heraus Prominenz erzeugen, wie etwa die Figur des prominenten Reporters belegt. Dies ist dann gewissermaßen eine intertextuelle Variante des Medienverbundes.

Das Fernseben ist in sich selbst ein solcher Medienverbund. Darum ist es zu dem Ort geworden, wo unablässig Prominenz erzeugt wird und zirkuliert. Und dadurch hat sich letztlich auch der Begriff der Prominenz selbst gewandelt. Mit dem Fernsehen kommt die Prominenz als solche zur Erscheinung. Das Wesen der Prominenz ist dadurch, wie man überspitzt formulieren könnte, sowohl einsichtiger als auch undurchsichtiger geworden. Wenn das Fernsehen ein Prominenz erzeugender Medienverbund ist, so folgt daraus nicht, dass regelmäßiges Auftauchen im Fernsehen an sich schon Prominenz ausmacht. Als Medienverbund funktioniert das Fernsehen nur, insoweit der Prominente dort als Prominenter auftaucht. So üben die ,Gesichter' des Mediums Fernsehen als einer Institution - die Nachrichtensprecher, die Fernsehansagerinnen früherer Zeiten usw. im Fernsehen zunächst einmal ihren Beruf aus, wie etwa auch die Regisseure, die Produzenten oder die Kameramänner, die man nicht sieht. Daher sind Werner Köpcke oder Dagmar Berghoff an ihrem Arbeitsplatz ebenso wenig prominent wie Uwe Seeler auf dem Fußballplatz oder Willy Brandt im Bundestag. Man kann das gewissermaßen schon daran sehen, dass sie so tun müssen, als wären sie nicht prominent. Prominent mögen Dagmar Berghoff oder Werner Köpcke sein, weil sie als Personen Gegenstand eines Diskurses in Fernsehzeitschriften, Illustrierten und sonstigen Klatschspalten sein können. Damit ist es also noch nicht das Fernsehen als Medienverbund, das sie prominent macht.

Für den Prominenz erzeugenden Diskurs in diesen anderen Medien (den Illustrierten, Fernsehzeitschriften usw.) ist zunächst einmal charakteristisch, dass der Gegenstand dieses Diskurses nicht unmittelbar an ihm zu partizipieren braucht. Dagmar Berghoff oder Uwe Seeler müssen nicht selber zu Wort kommen, wenn über sie geschrieben wird. Es kann ohne ihr Zutun etwas über sie enthüllt werden und ihnen etwas nachgesagt werden. Prominent werden sie überdies nur, insoweit nicht über ihre Tätigkeit am Arbeitsplatz oder über ihre Leistung gesprochen wird - das hymnische Lob einer schauspielerischen Leistung oder eines Mannschaftskapitäns macht den Betreffenden vielleicht berühmt, aber keineswegs prominent. Dass der Betreffende an einem Diskurs über seine eigene Leistung nicht unkommentiert teilnehmen kann, leuchtet ein. Er kann es aber, insofern das so genannte Informationsbedürfnis der Öffentlichkeit über die eigentliche Darbietung hinausgeht. Aus dieser Perspektive sind natürlich diejenigen, die im Fernsehen zu sehen sind, besonders prominenzgefährdet. Einen rein leistungsbezogenen Diskurs über die Darbietung wird es dort, wo das Fernsehen der Arbeitsplatz ist, kaum geben.

Die Standardversion der Teilnahme am Prominenz erzeugenden Diskurs ist das Interview. Schon die einfache Frage an einen Fußballspieler, wie er seine eigene Leistung oder das Spiel beurteile, setzt voraus, dass die Meinung des Betreffenden auch unabhängig von seiner Fähigkeit zur Selbstbeurteilung von Interesse ist: Man will eben wissen, was er selbst dazu sagt. Gleichwohl befindet sich der Angesprochene hier noch in 
der Position des Sachverständigen; die Rede bleibt streng auf die jeweilige Darbietung bezogen. An der kategorischen Unterscheidung zwischen der Position des Sachverständigen und der des Prominenten ist umso mehr festzuhalten, als sich dieser Unterschied in der heutigen Medienlandschaft zu verwischen scheint. Je weiter sich das Interview von diesem Kern des Sachverstands entfernt (weil es so viele prominente Sachverständige gibt und weil der Sachverstand ein Vorwand für das Auftreten des Prominenten werden kann), umso stärker arbeitet es mit an der Herstellung von Prominenz bzw. setzt diese voraus.

Formen des Interviews sind auch der Hauptmotor bei der Erzeugung von Prominenz im Fernsehen als Medienverbund. Wenn das Fernsehen als Medienverbund funktioniert, kommt im Fernsehen das Fernsehen sozusagen noch einmal vor. Das Fernsehen ist nicht nur ein Medium, das etwas sendet, sondern auch ein Ort. Und zwar ein Ort, der selbst auf Sendung geht. Man sieht diesen Ort überall, wo sich das Fernsehen einen Ort einrichtet - vor allem also im Fernsehstudio. Das Fernsehstudio ist einerseits ein Ort, von wo aus etwas gesendet wird, was wir sehen können, aber es ist zugleich auch ein Ort, wo etwas geschieht. Das scheint uns inzwischen so selbstverständlich, dass man es sich erst einmal wieder klar machen muss.

Insofern der Nachrichtensprecher nur zu uns spricht, gibt es kein Geschehen im Studio (oder genauer: jedes Geschehen wäre - wie etwa ein Versprecher - eine Art Unfall in der Übertragung der Sendung). Diese Auffassung war in der Frühzeit des Fernsehens herrschend. Jeder Sprecherwechsel war hier eine Art Umschalten. Den Unterschied zu Heute sieht man allein daran, dass sich der Moderator einer Nachrichtensendung nunmehr gegen Ende mit einem teilweise launigen Spruch an seinen Kollegen vom Sport zu wenden pflegt, um ihm das Wort zu erteilen: Die Kommunikation zwischen diesen beiden Kollegen ist ein Geschehen im Fernsehstudio. Wenn wir Leute sehen, die miteinander kommunizieren, nehmen wir sie (auch an ihrem Arbeitsplatz) - vereinfacht gesagt als Menschen wahr.

Die Programmsparte, die als Erste auf ein solches kommunikatives Geschehen gesetzt hat, war - kaum zufällig - die so genannte „Unterhaltung“. „Ist ja alles sichtbar, was wir hier machen“, erklärte Peter Frankenfeld am 4. Oktober 1958 in der Fernsehshow Viel Vergnügen. Das bezog sich nicht nur auf die Fernsehzuschauer, sondern zugleich - allerdings in anderer Weise - auf das Publikum im Saal. Die Anwesenheit des Saalpublikums und später des Studiopublikums verbürgt das Fernsehen als sichtbaren Ort des Geschehens (Niehaus 1991), in dem dann auch mit Unterhaltungen und anderen Gesprächsformen unterhalten werden kann.

In Peter Frankenfeld oder Hans-Joachim Kulenkampff bekam der Fernsehzuschauer Prominente zwar zu sehen, aber als Prominente auftreten konnten auch diese Herren erst, wenn sie im Fernsehen als Gäste an einem anderen Ort als ihrem angestammten Sendeplatz auftraten. Erst das Fernsehen, das sich Gäste einlädt, organisiert sich endgültig als Medienverbund. Diese Gäste sind zunächst einmal in der Regel absolute oder ausnahmsweise relative Personen der Zeitgeschichte. Später ist das Fernsehen freilich dazu übergegangen, auch unspezifische Nichtprominente als Gäste einzuladen. Wesentlich ist, dass das Fernsehen unter dieser Bedingung zu einem Ort, zu einer Einrichtung wird, die dem Gast durch bestimmte Formen der Adressierung und der Rede Prominenz zuschreibt. Das Fernsehen verleibt das Prädikat der Prominenz. Als Medienverbund fungiert das Fernsehen zugleich als eine Institution, die Prominenz zuerkennen kann. Daher geht es bei der Prominenz nicht um den Bekanntheitsgrad einer Person, sondern um die Zuschreibung von Prominenz durch die jeweiligen Vertreter der Institution Fernsehen, die als eine Art Kommunikator fungieren. 
Nur unter der Voraussetzung, dass Prominenz ein gleichsam „angeheftetes“ Prädikat ist, lässt sich verstehen, weshalb sie nicht als Eigenschaft aufgefasst werden kann, die dem Betreffenden „anhaftet“. Nur dies erklärt auch, warum immer wieder davon die Rede ist, dass es einen Status des Prominenten gibt (Rezzori 1965, S. 13; Peters 1996, S. 31). Schon Karl Kraus hatte Prominenz zu einer Art „Steuerklasse“ erklärt und es damit für wesentlich gehalten, dass der Prominente Mitglied einer fiktiven Gruppe und damit Effekt diskursiver Zuschreibungen ist. Aber erst der Medienverbund Fernsehen lässt die Prominenz als solche sichtbar zu Wort kommen. Auch wenn die Klatschgeschichten und die Indiskretionen der Sensationspresse weiter bestehen, kommt die Prominenz erst im Fernsehen „zu sich selbst“. Was außerhalb des Fernsehens wuchert, hat trotz aller Exklusivinterviews nicht den institutionellen Rang des Fernsehens, in dem der Prominente wirklich auftritt.

Der Prominente tritt als Mensch auf. Auch die Sensationspresse hat es ja auf die menschlichen Schwächen, auf die Unfälle und die Abwege, oder wenigstens auf den Alltag und den Umgang der Betroffenen abgesehen. Schon Gregor von Rezzori spricht in diesem Zusammenhang von der „menschlichen Note“ (Rezzori 1965, S. 42), die Hervorragenden den nicht Hervorragenden näher zu bringen habe. Auch Christus, der Mensch gewordene Gott, werde uns schließlich nahe gebracht durch den „Augenblick der Schwäche auf dem Ölberg“ (Rezzori 1965, S. 43). Im Fernsehen, wo die Prominenz sich selbst zur sichtbaren Darstellung bringt, braucht es ein derartiges Schicksal, ein derartiges Erleiden nicht. Es genügt, dass die Prominenten sich kommunizierend sichtbar als Menschen geben. Je mehr die hervorragenden Leistungen in den Hintergrund treten, desto mehr tritt die Prominenz in den Vordergrund. Die so genannte Medienprominenz, bei der von einer ,hervorragenden Leistung' im eigentlichen Sinne nicht mehr die Rede sein kann, da diese eben darin besteht, sich kommunizierend als Mensch zu geben, ist insofern nicht eine Abart, sondern die Reinform der Prominenz - Prominenz als Alltag.

Dass Prominenz einen Status bezeichnet, der dem Betreffenden im Fernsehen zugesprochen werden kann, erkennt man etwa daran, dass der prominente Gast vom jeweiligen Kommunikator auf eine bestimmte Weise begrüßt, eingeführt, angesprochen, befragt wird. Mit der Zuerkennung des Prominenten-Status sind außerdem eine Anzahl an kommunikativen Verpflichtungen und Berechtigungen verknüpft, die einer eingehenden Analyse wert wären. So darf der Prominente zwar über sich sprechen, aber ebenfalls nur auf eine bestimmte Weise. Während sich Nichtprominente in Talk-Shows als Ratsuchende und Hilfsbedürftige an das Fernsehen wenden können (Niehaus 2001), ist dies dem Prominenten als solchem nicht gestattet. Er hat seine etwaigen Probleme - sein Schicksal - stets in der Vergangenheit bewältigt. Er hat gegebenenfalls ein berufliches Tief überwunden, ist vom Alkohol losgekommen, konnte eine Trennung verwinden. Keineswegs wird die Welt der Prominenz und das Leben der Prominenten als störungsfrei und glücklich, als glanzvoll und unbeschwert dargeboten; es sind auch nicht die Schwierigkeiten und Hindernisse der Zukunft aus dem Wege geräumt. Der Prominente ist nur jemand, der in der Lage ist, sein Schicksal zu meistern, es in die Hand zu nehmen und hinzunehmen. Er hat Ja zu sich gesagt. So tritt er häufig selbst als Helfer auf, indem er ein Beispiel gibt. Der Prominente verlangt und begebrt nichts von der Institution, in der er als Gast zu Hause ist.

Man könnte sagen, dass prominent ist, wer als Prominenter eingeladen werden kann. Dies ist die einzige Art und Weise, wie man die Prominenz schließlich doch zu einer Art Eigenschaft erklären kann. Dem entspricht auch die Feststellung, ein Mann wie Jürgen W. Möllemann habe mit seinem Tod aufgehört, ein Prominenter zu sein, weil er nicht mehr als Prominenter zurückkommen kann. Allerdings ist den Betreffenden nicht un- 
bedingt anzusehen, ob sie diese Eigenschaft haben. So verfügt das Fernsehen über ein reiches Reservoir an halb vergessenen und gleichsam schlafenden Prominenten, die es bei Bedarf zu neuem Leben erwecken kann.

Wenn Prominenz nicht eine bloße Folge von Aufmerksamkeitsakkumulation ist, sondern eine Frage des Status, dann geht es in ihr tatsächlich immer auch um Anerkennung - aber eben nicht um die Anerkennung einer eigentlichen Leistung. Vielmehr stellen die Redeordnungen und die Inszenierungen, die das Auftreten von Prominenz umgeben, für sich bereits einen Akt der Anerkennung dar. Prominenz als solche impliziert Anerkennung. Das Fernsehen kann, weil es eine Institution ist, nur mit Subjekten sprechen, die es anerkennt. Alle kritischen Fragen, die etwa an einen Dieter Bohlen oder an einen Boris Becker gestellt werden könnten, haben schon deshalb eine vorgängige Anerkennung zur Grundlage, weil das Fernsehen ihn eingeladen hat und weil er die Einladung aus freien Stücken angenommen hat. Ein Kinderschänder und Mörder wie Marc Dutroux ist berühmt und berüchtigt. Er zieht ein überaus hohes Quantum Aufmerksamkeit auf sich, aber prominent ist er nicht. Die Anerkennung, die darin liegt, dass ihn die Institution Fernsehen als Gesprächspartner einlädt, kann ihm unmöglich zuteil werden. Man kann nicht dadurch prominent werden, dass man ein Verbrechen begeht, sondern höchstens, nachdem man ein Verbrechen bereut oder hinter sich gelassen hat. Peter-Jürgen Boock zum Beispiel, der an der Entführung und Ermordung des Arbeitgeberpräsidenten Schleyer beteiligte RAF-Terrorist, wurde nach siebzehn Jahren Haft entlassen und war dann, nachdem er als Autor eines Buches zu diesem Thema in Erscheinung getreten war, zu Gast bei Johannes B. Kerner (zusammen mit dem damaligen Justizminister Hans-Jochen Vogel, dem damaligen Innenminister Gerhart-Rudolf Baum und dem damaligen BKA-Kriminaldirektor Wolfgang Steinke). Er trat dort als eine Art Konvertit auf und bekundete sein Bedauern und seine Reue. Aber das heißt gerade nicht, dass das Fernsehen der Ort der Konversion wäre. Das wäre ein Ereignis Prominente sind aber nicht im Fernsehen, damit sich etwas ereignet. Wenn sie ein Ereignis produzieren, fallen sie aus der Rolle. Nichtprominente können im Fernsehen bereuen oder sich aussöhnen; Prominente können gegebenenfalls höchstens eine Reue bekunden oder zitieren, die schon vorher aktenkundig geworden und Voraussetzung für die Einladung gewesen ist. Dann können wir ihnen anerkennende Aufmerksamkeit spenden.

Die einfache Tatsache, dass das Medium Vertreter - Kommunikatoren - hat, die sich an Subjekte adressieren können, macht das Medium zur Institution. Indem das Fernsehen den Status der Prominenz verleiht und kommuniziert, stiftet es die Prominenten als Gruppe. Es kann freilich niemals feststehen oder festgestellt werden, wer zu dieser Gruppe gehört und wer nicht. Der König kann im Prinzip alle, die er zum Ritter geschlagen hat, herbeirufen. Bei der Prominenz ist das nicht möglich. Die Gruppe der Prominenten wird nur gestiftet, insofern sie sich als jeweils sichtbare Gruppe im und durch das Fernsehen aktualisiert. Man kann dann zum Beispiel sehen, dass Prominente neben Prominenten sitzen und dass sie sogar miteinander sprechen. Bei derartigen Gelegenheiten wird sichtbar, dass die Prominenten als solche einander gleichgestellt sind - Dolly Buster und Norbert Blüm, Verona Feldbusch und Alice Schwarzer, Boris Becker und Richard von Weizsäcker. Die Gemeinschaft der Prominenten beruht auf gegenseitiger Anerkennung (auch die Begegnungen von Prominenten auf Pressebällen, Empfängen und Verleihungen werden von der Berichterstattung des Fernsehens als egalitär ins Bild gesetzt), aber es ist die Gemeinschaft derer, die nichts gemein haben. Daher können sie auch gemeinsam interniert werden, in einem „Dschungel-Camp“, wo sie nur herausgeholt werden, wenn sie für sich in Anspruch nehmen, „etwas anderes“ zu sein, nämlich 
ein Star. Wo der Star hervortritt, verblasst die Umgebung. Der Status der Prominenz hingegen stiftet eine Umgebung von Prominenten.

\section{Neue Erscheinungsformen der Prominenz im Fernsehen}

Wird Prominenz, wenn man sie als Status auffasst, zu einem wohlabgegrenzten Phänomen? Dies ist nur bedingt der Fall. Zwar erlaubt es diese Verschiebung, die institutionelle Logik von Prominenz - sozusagen die Prozeduren ihrer Zuerkennung de iure - zu beschreiben, aber die Frage, wie Prominenz de facto entsteht, gerät damit aus dem Blick. Schließlich kann man einwenden, dass das Fernsehen eben als Prominente nur Leute einlädt und zu Prominenten erhebt, die an sich schon prominent sind. Sicherlich kann und will das Fernsehen nicht jeden Beliebigen als Prominenten einführen. Irgendwie muss der Betreffende schon Aufmerksamkeit erregt haben, muss etwa schon ab und an die Spalten der Presse gefüllt haben. Und gewiss lassen sich Auswahlkriterien benennen, warum dieser Fußballprofi und nicht jener, dieser Bundestagsabgeordnete und nicht jener in eine Gesprächsrunde eingeladen wird. Ein Jürgen W. Möllemann oder ein Daniel Cohn-Bendit sind allemal besser als ein Parteisoldat, weil sie schon prominent sind. Aber wie sind sie es geworden?

Diese Frage kann nur empirisch beantwortet werden und ist in gewisser Weise auch müßig. Sie führt nur zurück auf die Frage nach den Eigenschaften, die jemand mitbringen muss, um prominent zu werden, auf die es letztlich nur die tautologische Antwort eines erforderlichen Willens zur Prominenz und einer erforderlichen Fähigkeit zu dauerhafter Erregung von Aufmerksamkeit gibt. Peter Lückemeier listet in seinem Buch So werden Sie prominent ohne systematischen Anspruch auf: Prominenz durch Ausscheidung, durch Geburt, durch Heirat, durch Zufall und durch Leistung (Lückemeier 2003, S. 57-120). Es kann so oder auch anders kommen. Die institutionelle Logik der Prominenz setzt erst ein, wenn es so gekommen ist. Prominenz ist das, was von diesen Wurzeln gelöst ist.

Gregor von Rezzori, der - verborgen in seinem Idiotenfübrer durch die deutsche Gesellschaft - die wohl interessanteste und jedenfalls die kurzweiligste Theorie der Prominenz vorgelegt hat, spricht in diesem Zusammenhang von der "doppelten Prominenz" und bemüht als Beispiel hierfür Soraya, die durch ihre Heirat mit dem Schah per Zufall prominent geworden, nach ihrer Verstoßung aber prominent geblieben, ja „doppelt prominent" geworden ist ob „der menschlichen Note ihrer gynäkologischen Märtyrerschaft, noch hinzugefügt den Mangel an Scheu, sich darin augenfällig schauzustellen“ (Rezzori 1965, S. 55). Diese Doppelung ist ein Strukturmerkmal von Prominenz; sie ist es, die Rezzori zufolge bewirkt, dass die Prominenz ein Status ist, der nicht vergehen kann: Der Prominente kann „verarmen an Erfolg“, aber dann „wird er gewinnen die Noblesse des einstmals Reichen und nun Verarmten“; auch kann man zwar „seinen Grad an Prominenz in Zweifel ziehen, die Qualität der Erfolgsleistung, mit der er diesen Grad errungen hat: am Zustand seiner Prominenz wird das nichts ändern. [...] Denn was immer der einmal prominent Gewordene tut oder zu tun unterläßt: er ist als Prominenter, was er ist, und bleibt es auch“ (Rezzori 1965, S. 58f.). Diese Feststellungen datieren aus einer Zeit, bevor das Fernsehen die Talk-Show erfunden hatte, die auf genau dieser Logik beruht und sie zur Vollendung bringt. Denn sie ist der Ort oder der Schauplatz, an dem der Status der Prominenz immer neu dadurch verliehen wird, dass der Betreffende eingeführt wird als jemand, der nicht zum ersten Mal als Prominenter auftaucht. Wo es ein erstes Mal gibt, da wird es auch ein letztes Mal geben - denn dort gibt es einen Anlass, der sich verbraucht haben wird. 
Und damit hängt es zusammen, wenn die als Status aufgefasste Prominenz gleichwohl nur bedingt ein abgegrenztes Phänomen ist, denn den Anlass, der sich verbraucht haben wird, gibt es nicht nur de facto oft genug, er kann auch als solcher sichtbar werden, sobald er im Fernsehen selbst produziert wird. Dies betrifft zunächst den schon erwähnten Unterschied zwischen den relativen und den absoluten Personen der Zeitgeschichte. Das Fernsehen kann jederzeit ein bestimmtes Ereignis zum Anlass nehmen, um einen Betroffenen ins Fernsehen zu holen (das Opfer eines Zugunglücks, die Angehörigen eines unschuldig in den USA Inhaftierten usw.). Die als Betroffene Eingeladenen werden eben nicht als Prominente eingeladen. Wenn der Anlass jedoch eine größere Reichweite bekommt, können sich die Grenzen verwischen. Der ehemalige RAF-Terrorist Peter-Jürgen Boock beispielsweise ist ein solcher Grenzfall. Er hat aus seiner Ereignisbeteiligung ein Buch gemacht und ist damit ans Licht der Öffentlichkeit getreten. Bei Johannes B. Kerner erscheint er nun sowohl als Betroffener wie auch als eine Art Prominenter - ein Eindruck, der dadurch verstärkt wird, dass auch die geladenen Politiker als Betroffene der damaligen Geschehnisse berichten. Aber anders als Hans-Jochen Vogel kann sich Peter-Jürgen Boock nicht einfach in der nächsten Gesprächsrunde im Fernsehen zu einem Thema äußern, das mit seiner Vergangenheit nichts zu tun hat. Und im Grunde kann er auch kein zweites Buch schreiben. Nur für eine Weile kann man den Betroffenen und den Prominenten verwechseln.

Bei den Anlässen, die das Fernsehen selber schafft, sieht der Vorhof zur Prominenz anders aus. Hier handelt es sich natürlich nicht um Betroffene, die ein Ereignis ereilt hätte oder die in ein Geschehen verstrickt wären. Im Gegenteil. Das Fernsehen war bei den Betreffenden schon dabei, bevor sie schließlich als Gast im Fernsehen aufgetreten sind. Dies geschieht in verschiedenen neueren Sendeformaten. Eine Familie lebt eine Zeit in einem Schwarzwaldhaus wie vor hundert Jahren und wird von der Kamera begleitet. Nachdem dieses Leben in einem Mehrteiler gesendet worden ist, werden sie zu Herrn Beckmann eingeladen. Gleiches geschieht mit jenen sieben Nicht-Läufern, die innerhalb eines Jahres für den New York-Marathon fit gemacht werden. Die Betreffenden werden hier nicht mehr wie Nichtprominente eingeführt. Aber wiederum ist die Prominenz an den Anlass gebunden, es ist gleichsam eine $\mathrm{Als-Ob-Prominenz.} \mathrm{Sie} \mathrm{ist} \mathrm{befristet.} \mathrm{Das} \mathrm{er-}$ kennt man unter anderem daran, dass die Betreffenden auf die Frage nach ihrer Zukunft eine Antwort geben müssen, die nichts mit dem Anlass zu tun hat, aus dem sie in diesem Moment im Fernsehen zu Gast sind.

Anders verhält es sich bei den Formaten, die unter dem Oberbegriff „Prominent durch Ausscheidung" (Lückemeier 2003, S. 57) zusammengefasst werden können - wie etwa auf unspezifische und komplexe Weise die verschiedenen Staffeln von Big Brother und auf spezifische und geradlinige Weise Star Search auf SAT.1 und vor allem Deutschland sucht den Superstar auf RTL. Die Ausscheidung arbeitet hier mit dem Versprechen, dass der Sieger der Ausscheidung nicht nur ein Star sein wird, sondern sogar ein Superstar. Dies ist Peter Lückemeier zufolge natürlich ein „Etikettenschwindel“, da nur Leute wie Madonna oder Paul McCartney diese Bezeichnung verdienten (Lückemeier 2003, S. 57). Um solche Vergleiche geht es bei dieser Titulierung aber nicht, sondern um die internen Vergleiche mit den übrigen Kandidaten. Alexander, der erste Titelträger, ist „Superstar“, damit auch die übrigen Kandidaten als Stars bezeichnet werden können. Und entsprechend besagt auch „Prominent durch Ausscheidung“ nicht, dass der letzte Übriggebliebene der Prominenteste ist. Es war bekanntlich der Drittplatzierte Daniel Küblböck, der in dieser Hinsicht unbezweifelbar einen zweifelhaften Sieg davongetragen hat. Es geht in diesem Zusammenhang nicht um die Scheinfrage, ab wann die jeweils verbliebenen Kandidaten prominent genannt werden dürfen, 
und auch nicht um Mutmaßungen, wie lang sie in den Medien präsent sein werden. Interessant ist vielmehr, dass sich die Kandidaten auf der einen Seite auf Befragen eine Karriere wünschen dürfen, die sie zu Prominenten macht, dass sie aber in der jeweiligen Sendung gleichwohl - wie viele ihnen auch zujubeln mögen - ihren Status als Kandidat behalten (und der Kandidatenstatus ist natürlich die älteste Form, in der ein Nichtprominenter im Fernsehen auftreten kann). Daher werden sie innerbalb der Ausscheidung auch nicht als Prominente angesprochen. Mithin gilt auch die Anerkennung, mit der sie vor allem von den Juroren bedacht werden, ihnen als Kandidaten (sie ist graduiert und kontingent). Für die vom Publikum gespendete Anerkennung gilt das aber nicht. Solche Sendungen sind daher neuere Hybridformen, in denen Prominenz und Nichtprominenz einander überlagern.

Die Möglichkeit einer Überlagerung von Kandidatenstatus und Prominentenstatus lässt sich auch daran ersehen, dass das Sendeformat ebenso mit Prominenten zu funktionieren scheint. In der Comeback Show traten nicht zehn Unbekannte, sondern zehn Profis der Popmusik, die vor längerer Zeit Stars oder vor kürzerer Zeit beinahe Stars waren, als Kandidaten an und warben bei Juroren und Publikum um die Gunst eines diplomierten Comebacks. Dabei müssen sie auf der einen Seite als Kandidaten und auf der anderen Seite als Prominente angesprochen werden. Dieser Widerspruch wird auf zweifache Weise entschärft: Erstens setzt sich der Prominentenstatus schon deshalb durch, weil ja jeder Auftritt eines Prominenten im Fernsehen formal gesehen ein Comeback ist - eine Rückkehr an den Ort, wo sich die Prominenz verwirklicht. Und zweitens wird durch verschiedene Signale nahe gelegt, dass die Prominenten ihren Kandidatenstatus und die damit verbundene Prozedur der Ausscheidung spielerisch auffassen (schließlich haben sie ihr „Comeback“ gehabt, auch wenn sie nicht obsiegen).

Schon sehr früh hat das Fernsehen die Möglichkeit ergriffen, mit der Differenz zwischen Prominenz und Nichtprominenz zu spielen. So schien der Prominente auf der einen Seite aus einem ganz anderen Stoff als der Nichtprominente und auf der anderen Seite aus demselben Stoff zu sein. Lehrreich ist in dieser Hinsicht der Dauerbrenner der früheren Fernsehunterhaltung, das „heitere Beruferaten“ Was bin ich? mit Robert Lembke. In dieser Sendung musste das (aus vier Prominenten bestehende) Rateteam zunächst ausgefallene Berufe von drei Nichtprominenten und im Anschluss daran einen Prominenten erraten. Einerseits wurden die Nichtprominenten und der Prominente der gleichen Prozedur unterworfen (sie durften nur mit ja oder nein antworten usw.), andererseits wurde die Differenz schon dadurch betont, dass es nicht darum ging, den Beruf des Prominenten zu erraten, sondern ibn selbst - denn prominent ist jemand, von dem wir unterstellen dürfen, dass wir ihn erraten können, und der sich gleichsam selbst zum Beruf gemacht hat. Ins Bild gesetzt wurde die Differenz weiterhin durch die medientechnisch charmante Regel, dass die Mitglieder des Rateteams in dieser letzten Runde eine schwarze Augenbinde aufsetzen mussten und der Prominente keinen Laut von sich geben durfte - denn prominent ist jemand, den man sofort erkennt.

Eine beliebte Form, mit Nichtprominenten und Prominenten gleichermaßen zu spielen, ist das nicht weniger ehrwürdige Konzept der Candid Camera, der Versteckten Kamera. Hier wird der Prominente mit der Kamera dort beobachtet, wo er sich - wie unsereins - im Alltag wähnt. Aber die Alltagssituation wird auf die eine oder andere Weise denormalisiert, wodurch umgekehrt die „Medienprofessionalität auf ,Normalmaß““ (Friedrich 2001, S. 120) zurückgestutzt wird. In neuerer Zeit können sich die Prominenten aber auch freiwillig und wissentlich als Testpersonen melden und sich Aufgaben unterziehen, die für Nichtprominente erdacht worden sind. So gibt es beispielsweise in Abständen eine Spezialausgabe von Günther Jauchs Wer wird Millionär? mit 
Prominenten; oder die Prominenten unterziehen sich stellvertretend den sportlichen Übungen, mit denen Johannes B. Kerner die Frage Wie fit ist Deutschland? zu beantworten sucht.

Gerade in diesen neueren Formen der Überlagerung von Prominenz und Nichtprominenz tritt das Phänomen der Prominenz besonders klar in seiner Eigenartigkeit hervor. Gibt es eine genauere Erklärung darüber, was Prominente sind, als die räumliche Anordnung in der von Hape Kerkeling am 28. Mai 2004 moderierten Sendung Der große Deutsch-Test? Das Studiopublikum war hier Teilnehmer an einem Diktat und anderen Rechtschreibprüfungen. Es war (wie auch in anderen Sendungen dieses Typs) in Blöcke eingeteilt, die bestimmte Bevölkerungsgruppen repräsentierten: die Lehrer, die Schüler, die Chefs, die Sekretärinnen, die Landwirte, die Internationalen. Und vorne, auf eigens hergestellten Schulbänken: die Prominenten. Sie haben bei dem mitgemacht, was alle machen, und - wen wundert's? - mit vergleichbarem Erfolg. Als seien auch sie eine gesellschaftliche Gruppe. Tatsächlich handelt es sich bei den Prominenten um eine bloße Vergleichsgruppe, die für jeden Vergleich und für beliebige Aufgaben taugt. Neben dem unvermeidlichen Helmuth Karasek und dem ebenso unvermeidlichen Dirk Bach zählte auch der niedersächsische Ministerpräsident Christian Wulff dazu. Und Susan Stahnke, die von allen Prominenten am besten abgeschnitten hat. Wer hier die Schulbank drückt, hat einen Prominentenstatus, der ganz und gar unspezifisch bleibt. Wer hier die Schulbank drückt, empfiehlt sich für weitere Aufgaben.

\section{Literatur}

Determeyer, Ralf (1975): Personale Publizitätsdynamik. Massenmediale Modifikationen der bewußten und unbewußten Vermittlung des Menschen. Münster.

Franck, Georg (1998): Ökonomie der Aufmerksamkeit. Ein Entwurf. München/Wien.

Friedrich, Peter (2001): Von Spielleitern als Testleitern, Unfällen und Gesichtern in Fernsehshows - Verhaltensmikroskopie als Unterhaltungskunst. In: Rolf Parr, Matthias Thiele (Hg.): Gottschalk, Kerner und Co. Funktionen der Telefigur „Spielleiter“ zwischen Exzeptionalität und Normalität. Frankfurt am Main, S. 102-134.

Gerhards, Jürgen; Neidhardt, Friedhelm (1990): Strukturen und Funktionen moderner Öffentlichkeit. Discussion Paper FS III, S. 90-101. Berlin: Wissenschaftszentrum Berlin.

Kraus, Karl (1927/1961): Unsterblicher Witz. München.

Lückemeier, Peter (2003): So werden Sie prominent. Bergisch Gladbach.

Macho, Thomas H. (1993): Von der Elite zur Prominenz. Zum Strukturwandel politischer Herrschaft, in: Merkur 47, S. 762-769.

Münkler, Herfried (2004): Eilige Rückholaktion. Plädoyer für eine Resozialisierung der Elitevorstellungen in der Bundesrepublik. In: Frankfurter Rundschau online vom 2.2.2004.

Niehaus, Michael (1991): Das Fernsehen in seiner Sichtbarkeit. In: Wolfgang Tietze, Manfred Schneider (Hg.): Fernsehshows. Theorie einer neuen Spielwut. München, S. 105-132.

Niehaus, Michael (2001): „Action talking“ und „talking cure“. Der Spielleiter als Therapeut. In: Rolf Parr, Matthias Thiele (Hg.): Gottschalk, Kerner und Co. Funktionen der Telefigur „Spielleiter" zwischen Exzeptionalität und Normalität. Frankfurt am Main, S. 135-153.

Peters, Birgit (1993): Prominenz in der Bundesrepublik. Bedingungen und Bedeutungen eines Phänomens. Discussion Paper FS III, S. 92-103. Berlin: Wissenschaftszentrum Berlin.

Peters, Birgit (1996): Prominenz. Eine soziologische Analyse ihrer Entstehung und Wirkung. Opladen.

Rezzori, Gregor von (1965): Idiotenführer durch die deutsche Gesellschaft. Band 4: Prominenz. Vom Tun und Sein der Illüstren aus den Illustrierten. Ein Maßstab für das Übermaß des Mittelmaßes. Reinbek bei Hamburg.

Ricker, Reinhart (1989): Unternehmensschutz und Pressefreiheit. Heidelberg.

Seemann, Bruno (1996): Prominenz als Eigentum. Parallele Rechtsentwicklungen einer Vermark- 
tung der Persönlichkeit im amerikanischen, deutschen und schweizerischen Persönlichkeitsschutz. Baden-Baden.

Sieburg, Friedrich (1954): Von der Elite zur Prominenz. In: Die Zeit, vom 24.6.1954.

Traugott, Edgar (1979): Die Prominenz ist keine Elite. In: Gerd-Klaus Kaltenbrunner (Hg.): Rechtfertigung der Elite. Wider die Anmaßungen der Prominenz. Freiburg, S. 75-81. 